\title{
Research data communication strategy at the time of pandemics: a retrospective analysis of the Italian experience
}

\author{
Danilo Buonsenso ${ }^{1,2}$, Davide Pata ${ }^{2}$, Chiara Rocchetti ${ }^{3}$, Giampietro Marchetti ${ }^{4}$, Stefano Elia ${ }^{5}$, \\ Camillo Il Grande ${ }^{6}$, Piero Valentini ${ }^{1,2}$, Guido Levi ${ }^{3}$ \\ ${ }^{1}$ Department of Woman and Child Health, P. Universitario Gemelli IRCCS, Rome; ${ }^{2}$ Pediatrics Department, Università \\ Cattolica del Sacro Cuore, Rome; ${ }^{3}$ Interventional Pulmonology Department, ASST Spedali Civili, Brescia; \\ ${ }^{4}$ Pulmonology Department, ASST Spedali Civili, Brescia; ${ }^{5}$ Radiology Department, ASST Valcamonica, Esine (BS); \\ ${ }^{6}$ General and Laparoscopic Surgery Department, Ospedale Barone Romeo, Patti (ME), Italy
}

\begin{abstract}
Coronavirus pandemic has radically changed the scientific world. During these difficult times, standard peer-review processes could be too long for the continuously evolving knowledge about this disease. We wanted to assess whether the use of other
\end{abstract}

Correspondence: Dr. Guido Levi, Interventional Pulmonology Department, ASST Spedali Civili, P.le Spedali Civili 1, 25123 Brescia, Italy.

Tel. $+39.030 .3995593-$ Fax +39.030 .3995076 .

E-mail guido.levi@yahoo.it

Key words: Coronavirus; Covid-19; pandemic; peer-review; preprint repository.

Contributions: DB, study conceptualization, manuscript drafting; DP, data collection. All the authors performed data analysis and manuscript revision. All the authors have read and approved the final version of the manuscript and agreed to be accountable for all aspects of the work.

Conflict of interest: The authors declare that they have no conflict of interest.

Ethics approval: not applicable.

Acknowledgments: The authors wish to thank all "Coronavirus, SARS-CoV-2 e COVID-19 Gruppo per soli medici", "Pleural-Hub" and "Accademia di Ecografia Toracica" affiliates for their continuous support, interest and enthusiasm. Moreover, the authors wish to thank Mrs. R. Mancini and Dr. F. Levi for their invaluable support in the revision of the English language.

Informed consent:

Received for publication: 16 May 2021.

Accepted for publication: 13 July 2021.

${ }^{\circ}$ Copyright: the Author(s), 2021

Licensee PAGEPress, Italy

Monaldi Archives for Chest Disease 2022; 92:1931

doi: 10.4081/monaldi.2021.1931

This article is distributed under the terms of the Creative Commons Attribution Noncommercial License (by-nc 4.0) which permits any noncommercial use, distribution, and reproduction in any medium, provided the original author(s) and source are credited. types of network could be a faster way to disseminate the knowledge about Coronavirus disease. We retrospectively analyzed the data flow among three distinct groups of networks during the first three months of the pandemic: PubMed, preprint repositories (biorXiv and arXiv) and social media in Italy (Facebook and Twitter). The results show a significant difference in the number of original research articles published by PubMed and preprint repositories. On social media, we observed an incredible number of physicians participating to the discussion, both on three distinct Italian-speaking Facebook groups and on Twitter. The standard scientific process of publishing articles (i.e., the peer-review process) remains the best way to get access to high-quality research. Nonetheless, this process may be too long during an emergency like a pandemic. The thoughtful use of other types of networks, such as preprint repositories and social media, could be taken into consideration in order to improve the clinical management of COVID-19 patients.

\section{Introduction}

Since its first description [1], Severe Acute Corona Virus 2 Respiratory Syndrome (SARS-CoV-2) infection has rapidly spread all over the world being declared a pandemic by the World Health Organization on March 11, 2020 [2]. In this context clinicians who are directly involved in patients' care need to quickly gain access to all the possible knowledge on Coronavirus Disease 2019 (COVID-19) optimal management.

However, the standard scientific process that serves as a guide for science seems not prepared for such events changing so rapidly. Traditionally, researchers start from hypotheses, must obtain an institutional ethical committee approval, run the project, collect data, and finally write the paper. At this point, the submitted manuscript is evaluated by an editor who decides whether it is worth a peer-review process. In case of a positive first evaluation, days to weeks are needed for peer-review and weeks are required for the final approval. If accepted, not all papers are immediately available online. Meanwhile, an epidemic disease, such as COVID-19 is, may have infected and killed thousands of people and new preventive/diagnostic/therapeutic strategies implemented by a group would not be rapidly shared with other researchers.

During these difficult times, several networks [3] have launched open-source platforms for quick review of preprints research manuscripts. Some of these networks also allow comments among researchers. The time needed to post a research 
manuscript on these platforms is usually significantly shorter than posting it on PubMed, but the peer-review is lacking or is less accurate.

Not only preprint repository, but also social media are playing, for the first time in medical history, a primary role, giving the possibility to physicians worldwide to discuss clinical cases, share imaging, diagnostic or therapeutic experiences or local organization strategies.

\section{Methods}

We retrospectively collected and compared the data flow among three distinct groups of networks during the first three months of the pandemic: PubMed [4], one of the main search engines for scientific articles in the world, preprint repositories (such as biorXiv [5] and arXiv [6] networks) and social media in Italy, one of the countries that were most struck by the Coronavirus pandemic emergency in March 2020.

\section{Results}

On March 30, a PubMed search (using "COVID-19" [all fields] OR "Sars-CoV-2" [All fields]") retrieved 1908 items, whereas, on March 31, the biorXiv and arXiv networks have made 1057 and 234 COVID-19/SARS-CoV-2 preprints respectively available, since the first submission on January 13. Figure 1 shows the distribution of different article types from the three different databases per each month. In particular, the appearance of original studies on PubMed seems to be much slower than on the analyzed preprint repositories $(\mathrm{p}<0.001)$. Notably, the trend is different for case reports and other types of manuscript.

As Italy has been hit earlier and more strongly than other countries, three Italian-speaking Facebook groups have allowed physicians to virtually meet and discuss about clinical issues, traditional imaging (chest X-ray and CT scan) findings and imaging case discussion and lung ultrasound use in COVID-19.

The first group ("Coronavirus, SARS-CoV-2 e COVID-19 Gruppo per soli medici" - "Coronavirus, SARS-CoV-2 and COVID-19, physicians-only group" [7]) accepts only physicians. During the subscription proposal, users need to share their medical council number and a group of 28 administrators evaluate each request. In every post, each physician must state full name and surname and affiliation. During March 2020, 8677 posts $(+8754 \%$ compared with February), 309,516 comments $(+212 \%)$ and $1,400,000$ reactions $(+228 \%)$ were published. Initially created for Italian physicians only, many colleagues from several countries subscribed (Italy 86,843, United Kingdom 641, France 583, Switzerland 570, United States 461, top five represented countries). Here we witnessed the first experiences from Northern Italy about pronation in ventilated patients, or anti-interleukine- 6 drugs (such as tocilizumab, communicated on Facebook on March 6 by Prof. Ascienzo on his page [8]), or also the first report of a large necropsy series of COVID-19 patients performed in Brescia in a BL3 facility (unpublished data).

The Facebook group "Pleural-Hub" [9] has been actively discussing COVID-19 radiological cases. In March 2020, 522 posts (+668\% compared with February), 6474 comments (+485\%) and 18,500 reactions $(+556 \%)$ were published. Currently 4447 doctors ( $+36 \%$ compared with March 3, 2020) from several countries in the world subscribed (Italy 3901, United Kingdom 34, Greece 43, India 32, top five represented countries).

Italy has a great tradition of lung ultrasound, and a lot of physicians are actively sharing their ultrasound cases on the Facebook group "Accademia di Ecografia Toracica" ("Thoracic Ultrasound Academy") [10]. Lung ultrasound is being used both in the home care of COVID-19 patients and in those admitted to ICUs. Some explanatory videos of lung ultrasound findings are also available. During March 2020, 185 posts ( $+302 \%$ compared with February), 2897 comments $(+405 \%)$ and 8720 reactions $(+350 \%)$ had been published. Currently, 3233 doctors ( $+85 \%$ compared with March 3, 2020) subscribed (Italy 3014, United Kingdom 19, Germany 13, France and Spain 12 each, top five represented countries).

Additionally, on Twitter $\left.^{(}\right)$there is an impressive amount of information about COVID-19. Alkemy Lab ${ }^{\circledR}$ analyzed (using artificial intelligence methods) the main trends about COVID-19 [11]. In Italy only, between February 18, 2020 and March 05, 2020,

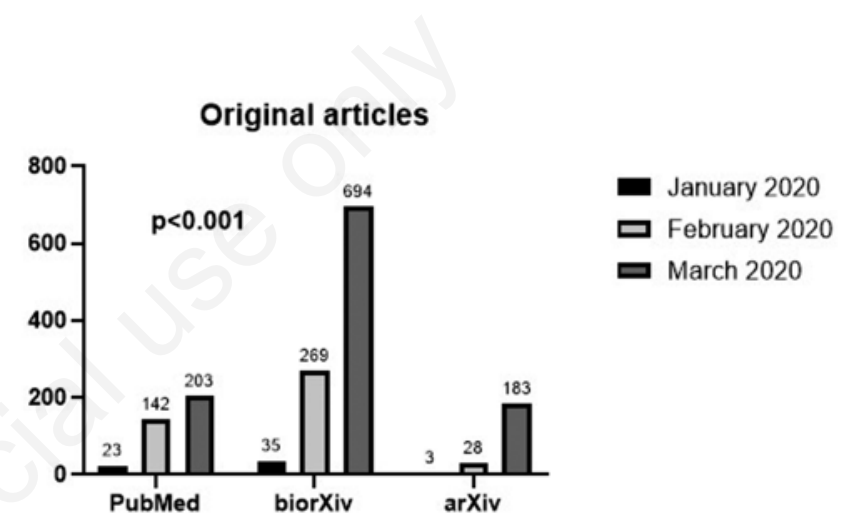

Case reports

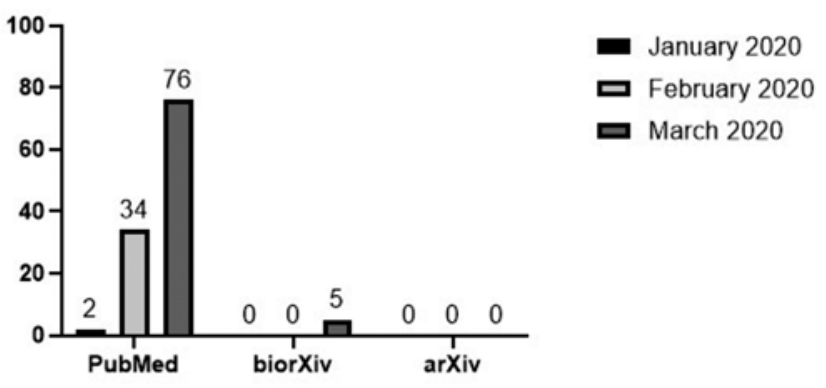

Other manuscripts

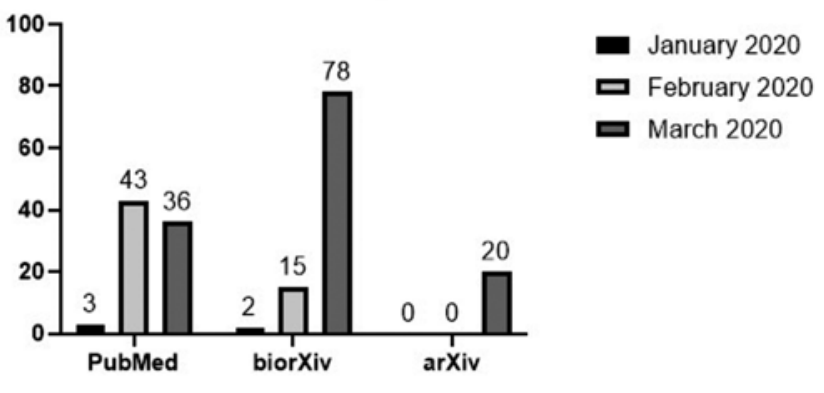

Figure 1. Publication of different article types (original studies, case series, others (comments, letters, etc.) from the three different databases (PubMed, bioRxiv, arXiv) in January, February and March 2020. 
2,225,689 items about Coronavirus had been published, consisting of many types of post (e.g., psychological issues, epidemiological analyses, imaging, case discussions, etc.). On March 31, 2020 we contacted one of the biggest healthcare social media analytics companies (Symplur ${ }^{\circledR}$, Los Angeles, CA, USA) in order to analyze the main topics about COVID-19 published on Twitter ${ }^{\circledR}$. The company replied that at that moment over 100 million tweets about Coronavirus were published every day, preventing any possible comprehensive analyses. To give an example, while searching for the hashtag \#POCUS, it is possible to watch several lung ultrasound videos of COVID-19 patients, including images from one of the most experienced researchers in the lung ultrasound field (i.e., dr. Volpicelli [12] currently involved in the front line in Northern Italy). Furthermore, on the same social media, public health experts share and comment main epidemiological data and analyses about national and international trends on COVID-19.

\section{Discussion}

Any news about COVID-19 on social media and preprint repositories are online, free, accessible everywhere in any moment to anyone.

However, the possible advantages of these new forms of data sharing are counterbalanced by the risk of diffusing lower quality information. Sumner et al. analyzed 535 preprint articles related to COVID-19 for eight transparency criteria and recorded study location and funding information [13]. They observed that researchers have lined up to help during this crisis, quickly facing important public health questions, often without funding or support from external organizations. However, most authors are not always following the best reporting practices. Many authors did not make their data readily accessible or did not share basic codes. While scientists are under significant pressure to promote their research, these findings suggest that the authors may not always recognize the importance of reproducibility criteria and/or the possible important role of these new forms of publication and dissemination. Researchers should improve their practices, but we could also need a better and quicker way to present urgently needed and useful data.

The COVID-19 outbreak is teaching healthcare workers a lot. However, it is also showing that the current method of sharing the scientific knowledge may be suitable for standard practice but could not be appropriate for emergency and unusual situations, such as the one we are still facing now. In countries with thousands of new cases per day, physicians need to learn something new every day. We, those actively involved in clinical practice and visiting patients, should not wait for the usual standard process of scientific knowledge, usually requiring several weeks; we currently need to improve our practice much more quickly. In this context, the whole research process may need a "revolution" involving institutions, researchers and journals.

Indeed, institutions could reduce the paperwork (currently too unsustainable and time-consuming for most researchers) and shorten time for Ethical Committees' approvals or even removing their obligatoriness in particular situations, such as data collection and sharing of anonymized clinical data for observational research during epidemics. Researchers are required to improve adhesion to high quality research principles, particularly if they want to upload preprints or quickly share data on social media, guaranteeing to provide high quality data. To give examples, this could result in providing ORCID ID, sharing raw data, providing a clear data availability statement and following the appropriate guidelines for data presentation. Journals should also take part in this process. Revisions are still made by peer-reviewers for free, and this can often take an extremely long time since researchers must review papers during free times after their own practice. The possibility to consider revision-fees might improve both the speed and the quality of revisions. Additionally, Publons ${ }^{\circledR}$ has recently started to collect reviews and to analyze the quality of revisions and this may ultimately improve their quality as well [14]. Journals could consider how to recognize reviewers' work: to give an example, revisions might be included in curriculum development for career purposes. Also, some journals started to use social media to develop active forum/networks and stimulate active case discussion. Nonetheless, scientific societies websites started to create interesting tools in order to make information as widely known as possible (e.g., the ERS COVID-19 resource center [15], and particularly the "COVID-19: Guidelines and recommendations directory", an international directory of guidelines and best practice recommendations prepared by ERS partner societies around the world focused on the management and care of COVID-19). Ultimately, social media are playing a growing role in our lives and clinicians' time is always shorter, thus we cannot miss the chance to take advantage of them.

\section{Conclusions}

COVID-19 is changing several aspects of our lives, including how we look for novel information, showing us that many common daily practices could be changed or improved. COVID-19 has also led us to think that the scientific community and research processes could change, as well.

Although the standard practice of publishing scientific articles on journals, after a careful peer-review process, remains the best way to gain access to high-quality research, the thoughtful use of preprint repositories and social media could be taken into consideration in order to improve the clinical management of patients during difficult times, such as during the COVID-19 pandemic.

\section{References}

1. Zhu N, Zhang D, Wang W et al. A novel coronavirus from patients with pneumonia in China, 2019. N Engl J Med 2020; 382:727-33.

2. World Health Organization. WHO Director-General's opening remarks at the media briefing on COVID-19 - 11 March 2020. Available from: https://www.who.int/director-general/speeches/detail/who-director-general-s-opening-remarks-at-themedia-briefing-on-covid-19-11-march-2020

3. Johansson MA, Saderi D. Open peer-review platform for COVID-19 preprints. Nature 2020;579:29.

4. PubMed.gov [Internet]. Available from: https://pubmed.ncbi. nlm.nih.gov/

5. biorXiv.org [Internet]. Available from: https://www.biorxiv.org/

6. arXiv.org [Internet]. Available from: https://arxiv.org/

7. Facebook [Internet]. Coronavirus, Sars-CoV-2 e COVID-19 gruppo per soli medici. Available from https://www.facebook. com/groups/Coronavirusmediciitaliani

8. Facebook [Internet] Dott. Paolo Antonio Ascierto - Oncologo e ricercatore italiano. Available from https://www.facebook. com/dottpaoloascierto 
9. Facebook [Internet]. Pleural-Hub group. Available from https://www.facebook.com/groups/pleural.hub

10. Facebook [Internet] Accademia di Ecografia Toracica group. Available from https://www.facebook.com/groups/ 1118364641543554

11. Alkemy Lab [Internet]. La Geo Intelligence del Lab per il COVID19. Available from: https://www.alkemylab.it/it/blog/ la-geo-intelligence-del-lab-per-il-covid19/

12. Volpicelli G, Elbarbary M, Blaivas M, et al. International evi- dence-based recommendations for point-of-care lung ultrasound. Intensive Care Med 2012;38:577-91.

13. Sumner JQ, Haynes L, Nathan S et al. Reproducibility and reporting practices in COVID-19 preprint manuscripts. medRixv 2020. 03.24.20042796

14. Publons [Internet]. Evaluation Reviews. Available from: https://publons.com/journal/6931/evaluation-review/

15. ERS COVID-19 resource center [Internet]. Available from: https://www.ersnet.org/covid-19/ 